\title{
Conservação de caqui 'Fuyu' com o tratamento em pré-colheita e pós-colheita com 1-metilciclopropeno
}

\author{
Marcelo José Vieira( ${ }^{(1)}$, Luiz Carlos Argenta(1), Cassandro Vidal Talamini do Amarante ${ }^{(2)}$, \\ Cristiano André Steffens ${ }^{(2)}$ e Edson Luiz de Souza ${ }^{(1)}$
}

\begin{abstract}
(1)Empresa de Pesquisa Agropecuária e Extensão Rural de Santa Catarina, Estação Experimental de Caçador, Caixa Postal 591, CEP 89500-000 Caçador, SC, Brasil. E-mail: marvieira@gmail.com, argenta@epagri.sc.gov.br, edsonluiz@epagri.sc.gov.br (2)Universidade do Estado de Santa Catarina, Centro de Ciências Agroveterinárias, Avenida Luiz de Camões, oo 2.090, CEP 88520-000 Lages, SC, Brasil. E-mail: cassandro.amarante@udesc.br, cristiano.steffens@udesc.br
\end{abstract}

Resumo - O objetivo deste trabalho foi comparar os efeitos da pulverização pré-colheita de 1-metilciclopropeno aquoso (1-MCPa) e tratamento pós-colheita de 1-metilciclopropeno gasoso (1-MCPg) sobre a maturação e a qualidade de caqui (Diospyros kaki) 'Fuyu'. Pulverizaram-se $150 \mathrm{mg} \mathrm{L}^{-1}$ de 1-MCPa nas plantas, na primeira semana de colheita comercial. Os frutos foram colhidos aos 1, 7, 14 e 21 dias, após a pulverização do 1-MCPa (DAAM), e tratados ou não com $0,6 \mu \mathrm{L} \mathrm{L}^{-1}$ de 1-MCPg, um dia após a colheita. Os tratamentos constituíram-se de controle, 1-MCPa, 1-MCPg e 1-MCPa+1-MCPg. Os frutos foram armazenados por 70 dias a $0^{\circ} \mathrm{C}$, em atmosfera modificada (AM), seguido por sete dias a $23^{\circ} \mathrm{C}$. O tratamento com 1-MCPa não afetou a maturação dos frutos na planta, mas reduziu o amolecimento prematuro de polpa e o distúrbio mancha translúcida em pós-colheita. A incidência de frutos moles após a armazenagem (média de três anos) foi superior a $84 \%$ no controle e inferior a $18 \%$ com 1-MCPg, independentemente da época de colheita, e de 15, 34, 40 e 75\%, quando os frutos foram tratados com 1-MCPa e colhidos 1, 7, 14 e 21 DAAM, respectivamente. A eficácia do 1-MCPa em frutos colhidos 1 DAAM é semelhante à do 1-MCPg e é reduzida com o atraso da colheita dos frutos a partir da data de sua aplicação.

Termos para indexação: Diospyros kaki, atmosfera modificada, distúrbios fisiológicos, etileno, fruto, dano por frio.

\section{Fruit quality preservation of 'Fuyu' persimmon by preharvest and postharvest treatments with 1-methylcyclopropene}

\begin{abstract}
The objective of this work was to compare the effects of preharvest spraying of aqueous 1-methylcyclopropene (1-MCPa) and of postharvest treatment of gaseous 1-methylcyclopropene (1-MCPg) on fruit maturity and quality of 'Fuyu' persimmon (Diospyros kaki). Spraying of $150 \mathrm{mg} \mathrm{L}^{-1} 1$-MCPa was done on trees in the first week of commercial harvesting. Fruit were harvested at 1, 7, 14, and 21 days after 1-MCPa spraying (DAMS), and treated or not with $0.6 \mu \mathrm{L} \mathrm{L}^{-1} 1-\mathrm{MCPg}$ one day after harvest. The treatments consisted of a control, 1-MCPa, 1-MCPg, and 1-MCPa+1-MCPg. Fruit were stored at $0^{\circ} \mathrm{C}$ for 70 days in modified atmosphere, followed by seven days at $23^{\circ} \mathrm{C}$. The treatment with $1-\mathrm{MCPa}$ did not affect fruit maturity on trees, but reduced the premature flesh softening and the occurrence of translucent stain disorder at postharvest. The incidences of soft fruit after storage (average of three years) were higher than $84 \%$ for the control, and lower than $18 \%$ for fruit treated with $1-\mathrm{MCPg}$, regardless of fruit harvesting date, and they were $15,34,40$, and $75 \%$ for fruit treated with 1-MCPa and harvested at 1, 7, 14, and 21 DAMS, respectively. The efficacy of 1-MCPa is equivalent to that of 1-MCPg for fruit harvested at 1 DAMS and its is reduced by delaying fruit harvest from the date of its application.
\end{abstract}

Index terms: Diospyros kaki, modified atmosphere, physiological disorders, ethylene, fruit, chilling injury.

\section{Introdução}

Várias estratégias têm sido adotadas comercialmente para evitar os efeitos indesejados do fito-hormônio etileno sobre a deterioração pós-colheita de caquis, notadamente a armazenagem dos frutos à baixa temperatura (MacRae, 1987; Neuwald et al., 2005). No entanto, a vida do caqui armazenado em ambiente refrigerado é limitada pelo desenvolvimento de manchas escuras sobre a epiderme (Argenta et al., 2009) e danos por frio (MacRae, 1987), especialmente quando os frutos são armazenados a temperaturas próximas de $5^{\circ} \mathrm{C}$ (Collins \& Tisdell, 1995).

A atmosfera modificada (AM), considerada uma das principais tecnologias complementares à refrigeração, reduz efetivamente a ocorrência de dano por frio em 
caqui (MacRae, 1987; Ben-Arie \& Zutkhi, 1992; Neuwald et al., 2005; Argenta et al., 2009). A aplicação de 1-metilciclopropeno gasoso $(1-\mathrm{MCPg})$, inibidor da ação do etileno, reduz a produção de etileno, retarda o amadurecimento e estende a vida pós-colheita de inúmeras espécies de frutos climatéricos (Watkins, 2006). Os efeitos benéficos do tratamento pós-colheita com 1-MCPg em caqui têm sido associados à prevenção do amolecimento da polpa (Salvador et al., 2004; Krammes et al., 2006; Argenta et al., 2009).

As aplicações de 1-MCPg são realizadas em ambiente hermético que, em nível comercial, normalmente correspondem à própria câmara de armazenagem. A depender da cultura (maçã, por exemplo), logo após a aplicação do 1-MCPg, tecnologias adicionais como a atmosfera controlada (AC) podem ser imediatamente estabelecidas. No entanto, para que sejam armazenados em AM, após a aplicação do 1-MCPg, os caquis devem ser removidos da câmara de tratamento para o fechamento das bolsas de plástico. Em experimento de armazenagem de caqui 'Fuyu', tratados com 1-MCPg sem o uso da AM, mostrou-se que a bolsa de plástico é essencial para o armazenamento destes frutos, e que os melhores resultados são obtidos pela combinação de ambas as tecnologias (1-MCPg + AM) (Argenta et al., 2009).

A pulverização com 1-metilciclopropeno em meio aquoso (1-MCPa), em maçãs (McArtney et al., 2008; Yuan \& Li, 2008; DeEll et al., 2010; Scolaro et al., 2015) e peras (Villalobos-Acuña et al., 2010), retarda a maturação e reduz a queda pré-colheita dos frutos. No entanto, esses efeitos são dependentes da cultivar, local, ano agrícola (ou safra) e estádio de maturação dos frutos. Além disso, foi relatada resposta diferencial do 1-MCPa, quando aplicado em pré e em pós-colheita (Varanasi et al., 2013). Exemplo disto ocorre em maçã 'Honeycrisp', em que o tratamento pós-colheita com 1-MCPg não teve efeito sobre a ocorrência do distúrbio escaldadura profunda, enquanto a pulverização pré-colheita de 1-MCPa reduziu esse distúrbio (DeEll et al., 2010). Em algumas culturas, o que inclui a maçã (Elfving et al., 2007; McArtney et al., 2008; Varanasi et al., 2013; Scolaro et al., 2015) e o kiwi (Manríquez \& Defilippi, 2011), o tratamento pré-colheita com 1-MCPa também pode promover aumento da conservação da qualidade pós-colheita dos frutos.

O objetivo deste trabalho foi comparar os efeitos da pulverização pré-colheita de 1-metilciclopropeno aquoso (1-MCPa) e tratamento pós-colheita de 1-metilciclopropeno gasoso (1-MCPg) sobre a maturação e qualidade de caqui (Diospyros kaki) 'Fuyu'.

\section{Material e Métodos}

O experimento foi conduzido em um pomar comercial de caqui 'Fuyu', localizado no Município de Rio das Antas, SC, a $26^{\circ} 53^{\prime} 55^{\prime \prime} \mathrm{S}, 51^{\circ} 04^{\prime} 28^{\prime \prime} \mathrm{W}$, à altitude de 830 m, em 2008, 2009 e 2010. Plantas uniformes de caqui foram pulverizadas ou não com 1-MCP em meio aquoso (1-MCPa, Harvista, Agrofresh Inc.), combinado ou não com a aplicação pós-colheita de 1-MCP gasoso (1-MCPg, EthylBloc, Agrofresh Inc.).

A pulverização ou não de caquizeiros 'Fuyu' com 1-MCPa ocorreu na primeira semana de colheita comercial, quando a maioria dos frutos apresentavam a coloração da epiderme predominantemente amarela (mais de 50\% amarela). O 1-MCPa foi aplicado com pulverizador costal, pressurizado com $\mathrm{CO}_{2}$, e equipado com bico tipo leque OC-12. Pulverizaramse aproximadamente $1200 \mathrm{~L} \mathrm{ha}^{-1}$ a $420 \mathrm{kPa}$ de uma emulsão composta de 1-MCPa [150 mg (i.a.) $\left.\mathrm{L}^{-1}\right] \mathrm{e}$ óleo mineral Assist (1\%, v/v).

Os frutos foram colhidos e acondicionados em caixas de plástico com $9 \mathrm{~kg}$ de capacidade, aos 1,7 , 14 e 21 dias após a aplicação do 1-MCPa (DAAM). Frutos de apenas uma planta por bloco e tratamento (sem e com 1-MCPa) foram amostrados em cada data de colheita, e cada planta foi amostrada uma única vez. Imediatamente após a colheita, os frutos foram transportados, à temperatura ambiente, para o Laboratório de Pós-Colheita da Estação Experimental da Empresa de Pesquisa Agropecuária e Extensão Rural de Santa Catarina (Epagri), localizado a $20 \mathrm{~km}$ do pomar. Em cada data de colheita, amostraramse aproximadamente 140 frutos por tratamento. Vinte frutos foram utilizados para a determinação da qualidade à colheita, e os frutos restantes (120) foram divididos em dois grupos de 60 frutos e destinados aos tratamentos pós-colheita.

Antes da armazenagem, 60 frutos de cada um dos tratamentos pré-colheita (sem e com 1-MCPa) foram tratados ou não com 1-MCPg $\left(0,6 \mu \mathrm{L} \mathrm{L}^{-1}\right)$, segundo método descrito por Krammes et al. (2005). Os tratamentos pós-colheita consistiram de: um controle, sem uso de 1-MCPa e 1-MCPg; 1-MCPa; 1-MCPg; e 1-MCPa+1-MCPg. Os frutos dos quatro 
tratamentos foram acondicionados em bolsas de plástico de polietileno de baixa densidade (PEBD) com $80 \mu \mathrm{m}$ de espessura, com as seguintes medidas de permeabilidade: $\mathrm{O}_{2}, 4.127 \mathrm{~mL} \mathrm{~m}{ }^{-2}$ por dia; $\mathrm{CO}_{2}$, $21.078 \mathrm{~mL} \mathrm{~m}^{-2}$ por dia; e $\mathrm{H}_{2} \mathrm{O}, 3,8 \mathrm{~g} \mathrm{~m}^{-2}$ por dia. As bolsas foram vedadas com amarrilho, para criar a condição de AM. Os frutos foram armazenados por 70 dias, em câmara experimental a $0 \pm 0,5^{\circ} \mathrm{C}$ e $80 \pm 5 \%$ de umidade relativa (UR). Após este período, as bolsas de plástico foram abertas, e os frutos foram transferidos para a câmara de maturação a $23 \pm 2^{\circ} \mathrm{C}$ e UR de $80 \pm 5 \%$, por sete dias.

À colheita e após a armazenagem, os frutos foram analisados quanto à coloração da epiderme, firmeza da polpa e teor de sólidos solúveis (SS). Após a armazenagem, os frutos também foram avaliados quanto à severidade de distúrbios, como superfície translúcida, estrias e pinta preta pequena.

A coloração da superfície dos frutos foi estimada visualmente, tendo-se atribuído notas de 3,0 (cor verde-amarelo) a 8,0 (cor laranja-vermelho), conforme descrito por Krammes et al. (2007). O teor de SS foi determinado no suco obtido da secção equatorial dos frutos, com espremedor tipo Champion (Plastaket Mgf), com refratômetro digital (Atago, Tóquio, Japão).

A análise da firmeza de polpa foi realizada em dois lados opostos da superfície de cada fruto, de onde a epiderme foi previamente removida, com um penetrômetro com ponteira de $8 \mathrm{~mm}$ de diâmetro (Güss, África do Sul). A análise da firmeza de polpa também foi realizada por meio do tato, para identificar os frutos, que foram classificados como firmes (1), meio moles (2) e moles (3). Foram considerados firmes os frutos de textura crocante, que não poderiam ser amassados e cuja epiderme não poderia ser rompida pela pressão dos dedos das mãos na região equatorial. Foram considerados meio-moles os frutos de textura "gel" (ausência de suco) e que poderiam ser rompidos pela pressão dos dedos polegar e indicador, mas que não se rompiam pela queda de uma altura de $30 \mathrm{~cm}$ (sobre bancada de granito). Foram considerados moles os frutos de textura aquosa, de epiderme facilmente rompida pela queda de uma altura de $30 \mathrm{~cm}$ e que sempre apresentaram firmeza de polpa inferior a 2,2 N, na determinação pelo penetrômetro.

Os distúrbios identificados como superfície translúcida, estrias e pinta preta pequena foram determinados pela análise visual de cada fruto, tendo- se atribuído notas de acordo com a área da superfície afetada como: ausência de dano; leve, menos de 5\% da superfície afetada; moderada, de 5 a $25 \%$ da superfície afetada; e severa, mais de $25 \%$ da superfície afetada.

O experimento foi realizado em blocos ao acaso, com quatro repetições, tendo sido a unidade experimental constituída de uma planta, em arranjo fatorial $3 \times 4 \times 4$, constituído de ano de produção (2008, 2009 e 2010), data de colheita $(1,7,14$ e 28 DAAM) e tratamentos (controle; 1-MCPa; 1-MCPg; e 1-MCPa+1-MCPg). Uma exceção ocorreu em 2008, em que o fator data de colheita constituiu-se de apenas dois níveis (1 e 14 DAAM).

Os dados foram submetidos à análise de variância dos fatores principais (ano, data de colheita $\mathrm{e}$ tratamento) e das interações dos fatores. As diferenças entre tratamentos foram determinadas pelo teste de Tukey, a 5\% de probabilidade. Modelos matemáticos de firmeza da polpa em função do tempo (dias), após a pulverização do 1-MCPa, foram determinados apenas no tratamento com 1-MCPa, por análise de regressão. Estes modelos foram usados para estimar o tempo entre a data de aplicação do 1-MCPa e a data em que os frutos atingiram firmeza de $20 \mathrm{~N}$ - firmeza crítica, abaixo da qual os frutos são considerados impróprios para a comercialização (Crisosto et al., 1999) -, durante a maturação pós-armazenagem, para 2009 e 2010. Modelos matemáticos de incidência de frutos moles em função do tempo (dias), após a pulverização do 1-MCPa, também foram determinados por análise de regressão e usados para estimar a incidência de frutos moles na data em que eles atingiram firmeza de $20 \mathrm{~N}$, para 2009 e 2010.

\section{Resultados e Discussão}

A evolução da maturação dos frutos na planta, evidenciada pela redução da firmeza de polpa, mudança da coloração (amarelecimento) da epiderme e aumento do teor de SS, apresentou diferenças entre os anos de avaliação e não foi afetada pela pulverização pré-colheita com 1-MCP em meio aquoso (1-MCPa) (Tabela 1).

Resultados semelhantes também foram observados em kiwi 'Hayward' por Manríquez \& Defilippi (2011). Em maçã, a pulverização com 1-MCPa proporcionou redução da concentração interna de etileno e retardamento da degradação do amido e da perda de 
firmeza de polpa (Elfving et al., 2007; McArtney et al., 2008; Yuan \& Li, 2008; Scolaro et al., 2015); assim, 1-MCPa representa uma ferramenta adicional para o manejo da colheita dos frutos.

Após o armazenamento em AM, seguido de sete dias de vida de prateleira, verificou-se que os tratamentos 1-MCPa, 1-MCPg e 1-MCPa+1-MCPg preveniram o amolecimento dos frutos, e quase a totalidade dos frutos-controle apresentavam-se moles, com firmeza de polpa próxima ao limite de detecção do penetrômetro $(\sim 0,9 \mathrm{~N})$ (Tabelas 2 e 3$)$. O rápido amolecimento dos frutos-controle, durante o período de prateleira, após a armazenagem, pode ser atribuído ao dano por frio (MacRae, 1987; Krammes et al., 2006; Argenta et al., 2009). O efeito do 1-MCP sobre a conservação da firmeza de polpa e redução da incidência de frutos moles foi influenciado pelo ano, pela data de colheita e forma de aplicação do produto. A máxima conservação da qualidade foi observada nos caquis tratados com 1-MCP na forma gasosa (1-MCPg) após a colheita. Frutos tratados com 1-MCPa e colhidos 1 DAAM apresentaram conservação da firmeza de polpa e redução da incidência de frutos moles semelhantes às de frutos tratados com 1-MCPg e 1-MCPa+1-MCPg, independentemente do ano. No entanto, observouse redução significativa do efeito do tratamento 1-MCPa sobre a conservação da firmeza de polpa, com o retardamento da colheita após a sua aplicação em campo (Tabela 2). O retardamento da colheita não afetou ou reduziu a incidência de frutos moles após a armazenagem, conforme o ano, para frutos tratados com 1-MCPg (Tabela 3). Ao contrário, o atraso da colheita não afetou ou aumentou a incidência de frutos moles nos frutos tratados com 1-MCPa (Tabela 3; Figura 1). A dinâmica da perda da eficiência do 1-MCPa pelo atraso na colheita dos caquis variou entre

Tabela 1. Firmeza de polpa, teor de sólidos solúveis, cor da epiderme (3-8) e severidade do dano de estrias (1-4) em caqui 'Fuyu', em três anos de avaliação e diferentes intervalos de colheita (dias), após a aplicação de 1-metilciclopropeno em meio aquoso $(1-\mathrm{MCPa})^{(1)}$.

\begin{tabular}{|c|c|c|c|c|c|c|c|c|}
\hline \multirow[t]{2}{*}{ Ano } & \multicolumn{2}{|c|}{$1 \mathrm{dia}$} & \multicolumn{2}{|c|}{7 dias } & \multicolumn{2}{|c|}{14 dias } & \multicolumn{2}{|c|}{21 dias } \\
\hline & Controle & 1-MCPa & Controle & 1-MCPa & Controle & 1-MCPa & Controle & 1-MCPa \\
\hline & \multicolumn{8}{|c|}{ Firmeza de polpa (N) } \\
\hline 2008 & $55,3 \mathrm{Aa}$ & $55,7 \mathrm{Ba}$ & - & - & $48,1 \mathrm{Bb}$ & $60,4 \mathrm{Aa}$ & - & - \\
\hline 2009 & $64,6 \mathrm{ABa}$ & $64,6 \mathrm{ABa}$ & $68,0 \mathrm{Aa}$ & $68,6 \mathrm{Aa}$ & $60,8 \mathrm{Ba}$ & $60,5 \mathrm{Ba}$ & $66,2 \mathrm{Aa}$ & $67,3 \mathrm{Aa}$ \\
\hline 2010 & $47,9 \mathrm{Ab}$ & $53,4 \mathrm{Aa}$ & $49,3 \mathrm{Aa}$ & $50,9 \mathrm{ABa}$ & $48,8 \mathrm{Aa}$ & $42,1 \mathrm{Cb}$ & $46,1 \mathrm{Aa}$ & $46,3 \mathrm{BCa}$ \\
\hline Média & 58,7 & 59,6 & 63,9 & 64,9 & 55,5 & 57,1 & 61,3 & 61,1 \\
\hline \multirow[t]{2}{*}{$\underline{\text { Desvio-padrão }}$} & 13,3 & 12,1 & 12,2 & 13,6 & 11,5 & 9,6 & 13,0 & 12,2 \\
\hline & \multicolumn{8}{|c|}{ Teor de sólidos solúveis $(\%)$} \\
\hline 2008 & $16,1 \mathrm{Ba}$ & $15,8 \mathrm{Aa}$ & - & - & $17,4 \mathrm{Aa}$ & $16,7 \mathrm{Aa}$ & - & - \\
\hline 2009 & $15,1 \mathrm{Ba}$ & $15,1 \mathrm{Ba}$ & $15,9 \mathrm{Ba}$ & $15,7 \mathrm{ABa}$ & $16,9 \mathrm{ABa}$ & $15,2 \mathrm{Bb}$ & $18,2 \mathrm{Aa}$ & $17,2 \mathrm{Aa}$ \\
\hline 2010 & $13,6 \mathrm{Cb}$ & $14,7 \mathrm{Aa}$ & $14,3 \mathrm{Bb}$ & $14,9 \mathrm{Aa}$ & $15,1 \mathrm{Aa}$ & $15,0 \mathrm{Aa}$ & $15,4 \mathrm{Aa}$ & $14,7 \mathrm{Ab}$ \\
\hline Média & 15,0 & 15,2 & 15,5 & 15,5 & 16,6 & 15,4 & 17,5 & 16,3 \\
\hline \multirow[t]{2}{*}{$\underline{\text { Desvio-padrão }}$} & 1,1 & 0,9 & 1,6 & 1,3 & 1,3 & 0,9 & 2,2 & 0,7 \\
\hline & \multicolumn{8}{|c|}{ Cor da epiderme $(3-8)^{(2)}$} \\
\hline 2008 & $6,0 \mathrm{Ba}$ & $6,1 \mathrm{Aa}$ & - & - & $6,5 \mathrm{Aa}$ & $5,5 \mathrm{Bb}$ & - & - \\
\hline 2009 & $3,6 \mathrm{Ba}$ & $3,6 \mathrm{Ba}$ & $4,9 \mathrm{Aa}$ & $4,9 \mathrm{Aa}$ & $4,9 \mathrm{Aa}$ & $4,4 \mathrm{Ab}$ & $5,3 \mathrm{Aa}$ & $4,7 \mathrm{Ab}$ \\
\hline 2010 & $3,9 \mathrm{Ba}$ & $4,1 \mathrm{Ba}$ & $4,1 \mathrm{Ba}$ & $4,2 \mathrm{Ba}$ & $4,3 \mathrm{Ba}$ & $4,2 \mathrm{Ba}$ & 4,9Aa & $5,2 \mathrm{Aa}$ \\
\hline Média & 4,6 & 4,6 & 4,7 & 4,8 & 5,2 & 4,8 & 5,2 & 4,8 \\
\hline \multirow[t]{2}{*}{ Desvio-padrão } & 1,6 & 1,7 & 1,1 & 1,1 & 1,4 & 1,0 & 1,1 & 0,7 \\
\hline & \multicolumn{8}{|c|}{ Severidade de estrias $(1-4)^{(3)}$} \\
\hline 2008 & $1,4 \mathrm{Ab}$ & $1,7 \mathrm{Aa}$ & - & - & $1,5 \mathrm{Aa}$ & $1,6 \mathrm{Aa}$ & - & - \\
\hline 2009 & $1,3 \mathrm{Ba}$ & $1,3 \mathrm{Ba}$ & $1,5 \mathrm{Aa}$ & $1,4 \mathrm{ABa}$ & - & - & $1,5 \mathrm{Aa}$ & $1,6 \mathrm{Aa}$ \\
\hline 2010 & - & - & - & - & - & - & - & - \\
\hline Média & 1,3 & 1,5 & 1,5 & 1,4 & 1,5 & 1,6 & 1,5 & 1,6 \\
\hline Desvio-padrão & 0,5 & 0,5 & 0,5 & 0,5 & 0,7 & 0,6 & 0,6 & 0,5 \\
\hline
\end{tabular}

${ }^{(1)}$ Médias seguidas por letras iguais, minúsculas nas linhas (tratamentos em uma mesma data de colheita) e maiúsculas nas colunas (datas de colheita em um mesmo tratamento), não diferem pelo teste de Tukey, a 5\% de probabilidade. ${ }^{(2)}$ Cor da epiderme: 3 , verde-amarelo; 8 , laranja-vermelho. ${ }^{(3)}$ Severidade do dano de estrias: 1 , ausência de dano; 2 , leve, menos de $5 \%$ da superfície afetada; 3 , moderada, de 5 a $25 \%$ da superfície afetada; e 4 , severa, mais de $25 \%$ da superfície afetada. 
anos (Figura 1). A colheita aos 7 DAAM resultou em perda da eficiência do $1-\mathrm{MCPa}$, quanto à conservação da textura, que foi maior em 2009 do que em 2010. No entanto, a perda da eficiência do $1-\mathrm{MCPa}$, em frutos colhidos aos 14 DAAM, não diferiu entre os três anos, apesar da menor firmeza da polpa e maior incidência de frutos moles em 2009 do que em 2008 e 2010 (Tabelas 2 e 3; Figura 1).

Em 2009, nas análises realizadas após o armazenamento refrigerado em frutos colhidos aos 7 , 14 e 21 DAAM, a firmeza de polpa de caquis tratados com 1-MCPa foi semelhante à de frutos-controle, embora a incidência de frutos moles tenha sido significativamente inferior aos 7 e 14 DAAM (Tabelas 2 e 3). No entanto, a firmeza de polpa de frutos tratados com 1-MCPa se manteve superior à de frutos-controle até 14 DAAM, em 2008 e 2010. Ressalta-se que, na colheita aos 14 DAAM em 2010, embora frutos tratados com 1-MCPa tenham apresentado firmeza de polpa superior à de frutos-controle, sua firmeza de polpa foi inferior ao limite de $20 \mathrm{~N}$ (Crisosto et al., 1999), e o percentual de frutos moles foi elevado (40\%). Aos 21 DAAM, em 2009 e 2010, frutos tratados com 1-MCPa não diferiram de frutos-controle quanto à firmeza de polpa e à incidência de frutos moles, o que indica perda total do efeito do 1-MCPa (Tabelas 2 e 3).

A firmeza de polpa dos frutos foi maior no tratamento 1-MCPa+1-MCPg, em comparação ao 1-MCPg, apenas nas colheitas aos 14 DAAM em 2008, aos 21 DAAM em 2009, e aos 7 DAAM em 2010 (Tabela 2). Estes resultados são indicativos de que a aplicação combinada 1-MCPa+1-MCPg não é superior à aplicação isolada do 1-MCPg. No entanto, em maçãs 'Golden Delicious', a combinação 1-MCPa+1-MCPg resultou em maior conservação da firmeza de polpa e menor produção de etileno do que o tratamento 1-MCPg (Varanasi et al., 2013). Interessante notar que, assim como observado em maçãs (McArtney et al., 2008), a perda progressiva dos efeitos do 1-MCPa, com o atraso da colheita após a aplicação, foi recuperada pela aplicação do 1-MCPg logo após a colheita.

Em geral, os consumidores preferem caquis 'Fuyu' firmes e, de acordo com Crisosto et al. (1999), caquis com firmeza de polpa inferior a $20 \mathrm{~N}$ são impróprios ao manuseio, distribuição e comercialização. Com base nessa observação, pode-se afirmar que, no presente estudo, o potencial de armazenagem dos caquis é inferior a 70 dias com refrigeração em AM, seguidos por mais sete dias a $23^{\circ} \mathrm{C}$, pois, os frutos-controle, nos três anos estudados, apresentaram média de firmeza de polpa inferior ao limite crítico de $20 \mathrm{~N}$ (Tabela 2). No entanto, frutos tratados com 1-MCPg ou com 1-MCPa+1-MCPg mantiveram a firmeza da polpa maior ou igual a $20 \mathrm{~N}$, depois da armazenagem durante 70 dias, mais sete dias a $23^{\circ} \mathrm{C}$, independentemente do ano e da data de colheita (Tabela 2). Em razão de a

Tabela 2. Firmeza da polpa $(\mathrm{N})$ em caqui 'Fuyu', após 70 dias de armazenagem em atmosfera modificada a $0^{\circ} \mathrm{C}$, mais sete dias a $23^{\circ} \mathrm{C}$, em três anos de avaliação e diferentes intervalos de colheita (dias), após a aplicação de 1-metilciclopropeno em meio aquoso (1-MCPa), em frutos tratados com 1-MCPa e 1-metilciclopropeno gasoso $(1-\mathrm{MCPg})^{(1)}$.

\begin{tabular}{|c|c|c|c|c|c|c|c|}
\hline \multirow[t]{2}{*}{ Ano } & \multirow[t]{2}{*}{ Tratamento } & \multicolumn{4}{|c|}{ Dias após a aplicação de 1-MCPa } & \multirow[t]{2}{*}{ Média } & \multirow[t]{2}{*}{ Desvio-padrão } \\
\hline & & 1 & 7 & 14 & 21 & & \\
\hline \multirow{4}{*}{2008} & Controle & $0,9 \mathrm{Bb}$ & - & $10,4 \mathrm{Da}$ & - & $6,5 \mathrm{C}$ & 10,1 \\
\hline & 1-MCPa & $48,6 \mathrm{Aa}$ & - & $25,0 \mathrm{Cb}$ & - & $35,0 \mathrm{~B}$ & 18,3 \\
\hline & 1-MCPg & $52,3 \mathrm{Aa}$ & - & $34,5 \mathrm{Bb}$ & - & $42,4 \mathrm{~A}$ & 14,4 \\
\hline & 1-MCPa+1-MCPg & $48,5 \mathrm{Aa}$ & - & $40,0 \mathrm{Ab}$ & - & $43,6 \mathrm{~A}$ & 12,8 \\
\hline \multirow{4}{*}{2009} & Controle & $0,9 \mathrm{Ba}$ & $0,9 \mathrm{Ba}$ & $0,9 \mathrm{Ba}$ & $1,1 \mathrm{Ca}$ & $0,9 \mathrm{C}$ & 0,3 \\
\hline & $1-\mathrm{MCPa}$ & $35,3 \mathrm{Aa}$ & $9,2 \mathrm{Bb}$ & $4,9 \mathrm{Bb}$ & $1,5 \mathrm{Cb}$ & $13,3 \mathrm{~B}$ & 22,8 \\
\hline & $1-\mathrm{MCPg}$ & $39,0 \mathrm{Ab}$ & $20,0 \mathrm{Ac}$ & $59,3 \mathrm{Aa}$ & $29,3 \mathrm{Bbc}$ & $37,0 \mathrm{~A}$ & 28,4 \\
\hline & 1-MCPa+1-MCPg & $34,9 \mathrm{Abc}$ & $24,2 \mathrm{Ac}$ & $66,3 \mathrm{Aa}$ & $37,5 \mathrm{Ab}$ & $40,8 \mathrm{~A}$ & 28,0 \\
\hline \multirow{4}{*}{2010} & Controle & $0,9 \mathrm{Ba}$ & $0,9 \mathrm{Da}$ & $0,9 \mathrm{Ca}$ & $0,9 \mathrm{Ba}$ & $0,9 \mathrm{C}$ & 0,0 \\
\hline & $1-\mathrm{MCPa}$ & $46,1 \mathrm{Aa}$ & $39,7 \mathrm{Cb}$ & $12,3 \mathrm{Bc}$ & $0,9 \mathrm{Bd}$ & $24,6 \mathrm{~B}$ & 22,2 \\
\hline & $1-\mathrm{MCPg}$ & $46,9 \mathrm{Aab}$ & $46,8 \mathrm{Bab}$ & $48,4 \mathrm{Aa}$ & $42,5 \mathrm{Ab}$ & $46,2 \mathrm{~A}$ & 11,4 \\
\hline & 1-MCPa+1-MCPg & $47,2 \mathrm{Ab}$ & $52,0 \mathrm{Aa}$ & $43,4 \mathrm{Abc}$ & $40,8 \mathrm{Ac}$ & $45,8 \mathrm{~A}$ & 10,4 \\
\hline
\end{tabular}

${ }^{(1)}$ Médias seguidas por letras iguais, em cada ano, maiúsculas nas colunas e minúsculas nas linhas, não diferem pelo teste de Tukey, a $5 \%$ de probabilidade. 
conservação da firmeza da polpa dos caquis tratados com 1-MCPa ter diminuído com a colheita tardia, estimou-se o tempo máximo de atraso da colheita a partir da data da pulverização, para que eles tivessem firmeza maior ou igual a $20 \mathrm{~N}$ depois da armazenagem, mais sete dias a $23^{\circ} \mathrm{C}$ (Figura 1 ).

A análise de regressão indicou que frutos tratados com 1-MCPa atingiriam a firmeza de polpa de 20 $\mathrm{N}$, quando colhidos aos 4 e 12 DAAM, em 2009 e 2010, respectivamente. No entanto, em 2008, os frutos tratados com 1-MCPa mantiveram firmeza de polpa acima do limite crítico, e a incidência de frutos moles nesse tratamento foi inferior a $6 \%$, mesmo quando colhidos aos 14 DAAM (Figura 1). Em 2009, a incidência de frutos moles no tratamento 1-MCPa foi alta $(30,8 \%)$, embora inferior à de frutos-controle $(84,8 \%)$, mesmo para a colheita realizada 1 DAAM (Tabela 3).

Em geral, o tratamento com 1-MCPa e 1-MCPg controlou (2010) ou pelo menos reduziu (2008 e 2009) a incidência do distúrbio mancha translúcida durante o período de prateleira, após o armazenamento em AM (Tabela 4). Estes resultados estão de acordo com Krammes et al. (2005) e Argenta et al. (2009), que observaram redução da incidência de mancha translúcida em caqui 'Fuyu' tratado com 1-MCPg. $\mathrm{O}$ efeito do $1-\mathrm{MCPa}$ sobre as manchas translúcidas foi semelhante ao do 1-MCPg em frutos colhidos
1 DAAM, e menor que o 1-MCPg em frutos colhidos 21 DAAM, em 2009, e em frutos colhidos 14 e 21 DAAM em 2010.

O desenvolvimento de estrias ocorreu durante todo o período de avaliação e, em geral, o tratamento com 1-MCPa e 1-MCPg não afetou a manifestação do distúrbio (Tabela 5), o que confirma resultados obtidos em estudos anteriores, que mostram ausência de efeito do 1-MCPg no controle do distúrbio (Krammes et al., 2006; Argenta et al., 2009). A presença de estrias também foi observada no momento da colheita, nas análises realizadas em 2008 e 2009, e não houve diferenças entre frutos-controle ou tratados com 1-MCPa (Tabela 1), o que indica que a indução do dano pode estar relacionada à existência de fatores pré-colheita não dependentes do etileno.

$\mathrm{O}$ tratamento com 1-MCPa e 1-MCPg não afetou a manifestação do distúrbio pinta preta (Tabela 6). Segundo Argenta et al. (2009), a incidência de pinta preta varia com o pomar e, em geral, o tratamento com 1-MCPg não altera significativamente a incidência desse dano. Biton et al. (2014) reportaram aumento da suscetibilidade à pinta preta com o atraso da colheita em caqui 'Triumph'. Segundo esses autores, simultaneamente ao amadurecimento, há um aumento do tamanho de microfissuras sobre a epiderme dos frutos, as quais favorecem a colonização e o desenvolvimento do fungo Alternaria alternata. Aliado

Tabela 3. Incidência (\%) de caqui 'Fuyu' mole, após 70 dias de armazenagem em atmosfera modificada a $0^{\circ} \mathrm{C}$, mais sete dias a $23^{\circ} \mathrm{C}$, em três anos de avaliação e diferentes intervalos de colheita (dias), após a aplicação do 1-metilciclopropeno em meio aquoso (1-MCPa), em frutos tratados com 1-MCPa e 1-metilciclopropeno gasoso (1-MCPg) ${ }^{(1)}$.

\begin{tabular}{|c|c|c|c|c|c|c|c|}
\hline \multirow[t]{2}{*}{ Ano } & \multirow[t]{2}{*}{ Tratamento } & \multicolumn{4}{|c|}{ Dias após a aplicação de 1-MCPa } & \multirow[t]{2}{*}{ Média } & \multirow[t]{2}{*}{ Desvio-padrão } \\
\hline & & 1 & 7 & 14 & 21 & & \\
\hline \multirow{4}{*}{2008} & Controle & $98,4 \mathrm{Aa}$ & - & $26,6 \mathrm{Ab}$ & - & $57,4 \mathrm{~A}$ & 38,9 \\
\hline & 1-MCPa & $4,5 \mathrm{Ba}$ & - & $5,4 \mathrm{Ba}$ & - & $5,1 \mathrm{~B}$ & 5,4 \\
\hline & 1-MCPg & $0,0 \mathrm{Ba}$ & - & $0,0 \mathrm{Ba}$ & - & $0,0 \mathrm{~B}$ & 0,0 \\
\hline & 1-MCPa+1-MCPg & $0,0 \mathrm{Ba}$ & - & $0,0 \mathrm{Ba}$ & - & $0,0 \mathrm{~B}$ & 0,0 \\
\hline \multirow{4}{*}{2009} & Controle & $84,8 \mathrm{Aa}$ & $98,2 \mathrm{Aa}$ & $100,0 \mathrm{Aa}$ & $78,3 \mathrm{Aa}$ & $90,4 \mathrm{~A}$ & 17,6 \\
\hline & 1-MCPa & $30,7 \mathrm{Bb}$ & $56,1 \mathrm{Bab}$ & $76,2 \mathrm{Ba}$ & $56,5 \mathrm{ABab}$ & $54,9 \mathrm{~B}$ & 20,1 \\
\hline & 1-MCPg & $15,4 \mathrm{Ba}$ & $17,5 \mathrm{Ca}$ & $1,7 \mathrm{Ca}$ & $9,2 \mathrm{BCa}$ & $10,9 \mathrm{C}$ & 8,9 \\
\hline & 1-MCPa+1-MCPg & $10,9 \mathrm{Bab}$ & $23,3 \mathrm{Ca}$ & $0,0 \mathrm{Cb}$ & $3,3 \mathrm{Cb}$ & $9,4 \mathrm{C}$ & 11,0 \\
\hline \multirow{4}{*}{2010} & Controle & $100,0 \mathrm{Aa}$ & $96,8 \mathrm{Aa}$ & $100,0 \mathrm{Aa}$ & $100,0 \mathrm{Aa}$ & $99,2 \mathrm{~A}$ & 2,7 \\
\hline & 1-MCPa & $8,6 \mathrm{Bc}$ & $12,7 \mathrm{Bc}$ & $40,0 \mathrm{Ba}$ & $95,0 \mathrm{Aa}$ & $39,1 \mathrm{~B}$ & 36,5 \\
\hline & 1-MCPg & $14,1 \mathrm{Ba}$ & 7,9BCab & $0,0 \mathrm{Cb}$ & $3,3 \mathrm{Bb}$ & $6,3 \mathrm{C}$ & 7,8 \\
\hline & 1-MCPa+1-MCPg & $13,1 \mathrm{Ba}$ & $0,0 \mathrm{Cb}$ & $0,0 \mathrm{Cb}$ & $0,0 \mathrm{Bb}$ & $3,3 \mathrm{C}$ & 29,2 \\
\hline
\end{tabular}

${ }^{(1)}$ Médias seguidas por letras iguais, dentro de cada ano, maiúsculas nas colunas e minúsculas nas linhas, não diferem entre si, pelo teste de Tukey, a $5 \%$ de probabilidade. 
a isso, o excesso de umidade (precipitação, irrigação e orvalho) próximo da época de colheita também favorece o desenvolvimento de pinta preta e, nestes casos, os sintomas podem ser observados no pomar.

O desenvolvimento de danos por frio, manifestados pelo amolecimento prematuro e manchas translúcidas, são os principais fatores que limitam o potencial de armazenagem de caqui 'Fuyu' (MacRae, 1987; Argenta et al., 2009). O desenvolvimento desses distúrbios em caqui 'Fuyu' é efetivamente inibido pela associação de 1-MCPg e AM (Argenta et al., 2009). Com o presente estudo, mostra-se que o tratamento com 1-MCPa aplicado antes da colheita pode ser tão eficaz quanto o 1-MCPg aplicado após a colheita, na prevenção desses distúrbios, a depender da época de colheita. O tratamento dos caquis com 1-MCPa antes da colheita é vantajoso em relação ao tratamento com 1-MCPg, por reduzir custos operacionais de carregamento, descarregamento e recarregamento da câmara de armazenagem com frutos, para tratamento com 1-MCPg, embalagem em AM e armazenagem refrigerada, respectivamente. No entanto, a eficiência do 1-MCPa na conservação pós-colheita foi reduzida em relação ao $1-\mathrm{MCPg}$, com o atraso da colheita por 7 a 21 dias, a partir da data de sua pulverização nas plantas (Tabelas 2 e 3), assim como observado em maçã (Elfving et al., 2007; McArtney et al., 2008) e pera (Villalobos-Acuña et al., 2010).

A ausência de efeitos aditivos dos tratamentos 1-MCPa e 1-MCPg, em frutos colhidos 1 DAAM, é um indicativo de que a maioria dos receptores de etileno - se não todos - estaria bloqueada pelo 1-MCPa aplicado antes da colheita. Em caquis 'Fuyu' (Krammes et al., 2005) e 'Rojo Brillante' (Salvador et al., 2004), a saturação de respostas ao 1-MCPg ocorre em concentração próxima ou ligeiramente superior a $0,1 \mu \mathrm{L} \mathrm{L}^{-1}$. Portanto, a dose de 1-MCPa aplicada em campo (150 $\mathrm{m} \mathrm{L}^{-1}$ ) pode ter proporcionado a saturação de resposta no fruto, correspondente à dose de $1-\mathrm{MCPg}$ aplicado em pós-colheita $\left(0,6 \mu \mathrm{L} \mathrm{L}^{-1}\right)$, o que retardou, assim, a perda de firmeza de polpa durante e após o armazenamento, em frutos colhidos imediatamente após a aplicação de 1-MCPa.

A inibição transitória da ação do etileno pelo 1-MCPg, observada em algumas espécies de frutos, e quando aplicado em baixas concentrações, tem sido atribuída à síntese de novos receptores de etileno (Sisler \& Serek, 1997; Pirrung et al., 2008). Da mesma forma, a reaquisição da suscetibilidade ao dano por frio, pelos caquis que permaneceram na planta por $7,14 \mathrm{ou}$

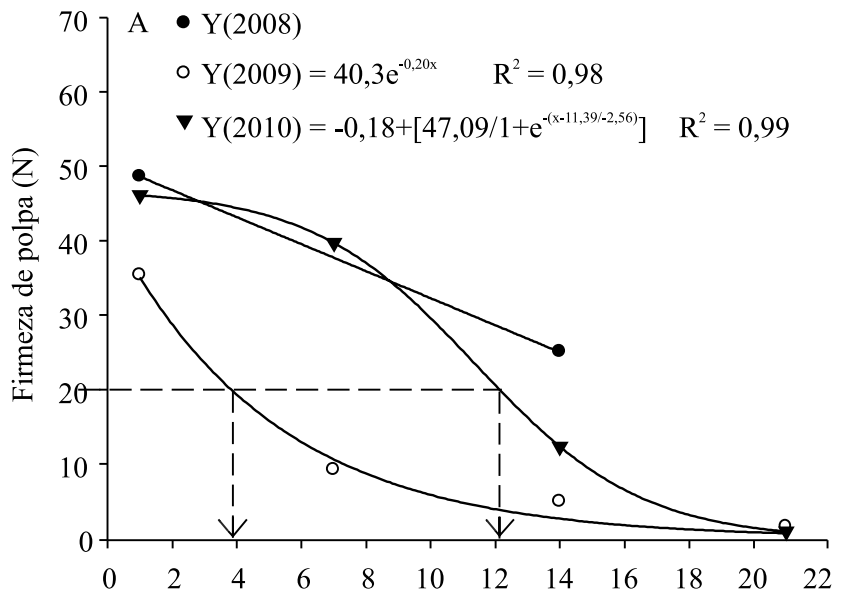

Dias após a aplicação do 1-MCPa

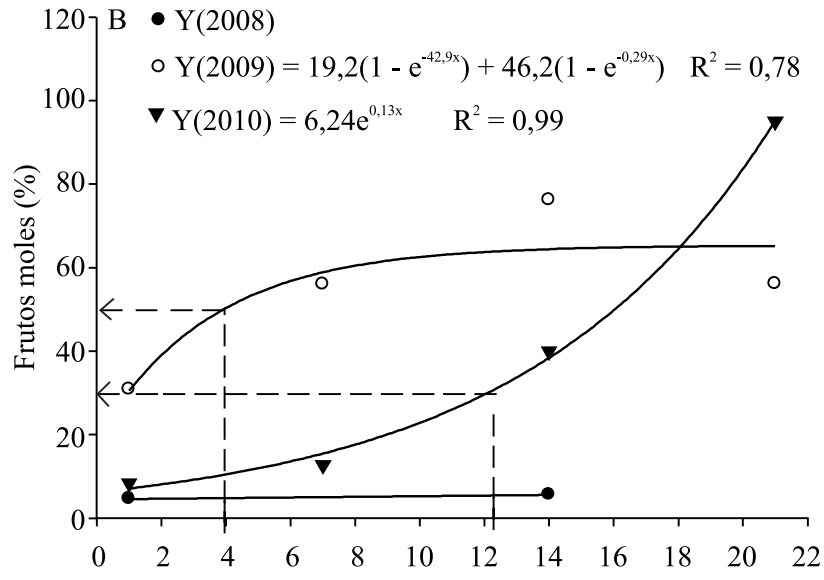

Dias após a aplicação do 1-MCPa

Figura 1. Firmeza de polpa e incidência de frutos moles em caqui 'Fuyu', após 70 dias de armazenagem em atmosfera modificada a $0^{\circ} \mathrm{C}$, mais sete dias a $23^{\circ} \mathrm{C}$, em três anos de avaliação e diferentes intervalos de colheita (dias), após a aplicação do 1-MCPa (1-meticilopropeno em meio aquoso). A, setas representam estimativas dos dias de colheita após a aplicação do 1-MCPa, para os frutos com firmeza de polpa de $20 \mathrm{~N}$. B, setas representam estimativas da incidência de frutos moles, para os frutos colhidos nos tempos estimados, com firmeza de polpa de $20 \mathrm{~N}$ após a armazenagem. 
21 DAAM, pode ser atribuída ao aumento progressivo da sensibilidade dos frutos ao etileno, em consequência da síntese de novos receptores. A maior duração dos efeitos do 1-MCPg, bem como do 1-MCPa (em frutos colhidos no dia seguinte à aplicação), durante a armazenagem refrigerada, possivelmente está relacionada, em grande parte, à menor taxa de síntese de novos receptores de etileno, decorrentes da baixa temperatura, baixa concentração de $\mathrm{O}_{2}$ e alta concentração de $\mathrm{CO}_{2}$ da $\mathrm{AM}$ induzida pelas bolsas de plástico.

Embora o 1-MCPa tenha sido tão eficiente quanto o 1-MCPg em frutos colhidos 1 DAAM, a concentração

Tabela 4. Severidade do dano de mancha translúcida (1-4) $)^{(1)}$ em caqui 'Fuyu', após 70 dias de armazenagem em atmosfera modificada a $0^{\circ} \mathrm{C}$, mais sete dias a $23^{\circ} \mathrm{C}$, em três anos de avaliação e diferentes intervalos de colheita (dias), após a aplicação do 1-metilciclopropeno em meio aquoso (1-MCPa), em frutos tratados com 1-MCPa e 1-metilciclopropeno gasoso (1-MCPg) ${ }^{(2)}$.

\begin{tabular}{|c|c|c|c|c|c|c|c|}
\hline \multirow[t]{2}{*}{ Ano } & \multirow[t]{2}{*}{ Tratamento } & \multicolumn{4}{|c|}{ Dias após a aplicação de 1-MCPa } & \multirow[t]{2}{*}{ Média } & \multirow[t]{2}{*}{ Desvio-padrão } \\
\hline & & 1 & 7 & 14 & 21 & & \\
\hline \multirow{4}{*}{2008} & Controle & $2,8 \mathrm{Aa}$ & - & $1,8 \mathrm{Ab}$ & - & $2,2 \mathrm{~A}$ & 0,72 \\
\hline & 1-MCPa & $1,1 \mathrm{Bb}$ & - & $1,4 \mathrm{Ba}$ & - & $1,3 \mathrm{C}$ & 0,49 \\
\hline & 1-MCPg & $1,0 \mathrm{Bb}$ & - & $1,8 \mathrm{Aa}$ & - & $1,5 \mathrm{~B}$ & 0,56 \\
\hline & 1-MCPa+1-MCPg & $1,1 \mathrm{Bb}$ & - & $1,2 \mathrm{Bb}$ & - & $1,2 \mathrm{C}$ & 0,38 \\
\hline \multirow{4}{*}{2009} & Controle & $2,9 \mathrm{Aa}$ & $2,8 \mathrm{Aa}$ & $2,9 \mathrm{Aa}$ & $2,8 \mathrm{Aa}$ & $2,9 \mathrm{~A}$ & 0,63 \\
\hline & 1-MCPa & $2,0 \mathrm{Bb}$ & $1,8 \mathrm{BCb}$ & $2,2 \mathrm{Bb}$ & 2,9Aa & $2,2 \mathrm{~B}$ & 0,96 \\
\hline & 1-MCPg & $1,7 \mathrm{Bb}$ & $2,2 \mathrm{Ba}$ & $1,9 \mathrm{Bab}$ & $1,9 \mathrm{Bab}$ & $1,9 \mathrm{C}$ & 0,85 \\
\hline & 1-MCPa+1-MCPg & $1,7 \mathrm{Bb}$ & $1,5 \mathrm{Cbc}$ & $1,3 \mathrm{Cc}$ & $2,2 \mathrm{Ba}$ & $1,7 \mathrm{D}$ & 0,77 \\
\hline \multirow{4}{*}{2010} & Controle & $1,5 \mathrm{Ab}$ & $1,6 \mathrm{Ab}$ & $2,4 \mathrm{Aa}$ & $2,4 \mathrm{Aa}$ & $2,0 \mathrm{~A}$ & 0,78 \\
\hline & 1-MCPa & $1,0 \mathrm{Bc}$ & $1,0 \mathrm{Bc}$ & $1,4 \mathrm{Bb}$ & $1,9 \mathrm{Ba}$ & $1,3 \mathrm{~B}$ & 0,55 \\
\hline & 1-MCPg & $1,0 \mathrm{Ba}$ & $1,0 \mathrm{Ba}$ & $1,0 \mathrm{Ca}$ & $1,0 \mathrm{Ca}$ & $1,0 \mathrm{C}$ & 0,00 \\
\hline & 1-MCPa+1-MCPg & $1,0 \mathrm{Ba}$ & $1,0 \mathrm{Ba}$ & $1,0 \mathrm{Ca}$ & $1,0 \mathrm{Ca}$ & $1,0 \mathrm{C}$ & 0,00 \\
\hline
\end{tabular}

${ }^{(1)}$ Severidade do dano de mancha translúcida: 1 , ausência de dano; 2 , leve, menos de $5 \%$ da superfície afetada; 3 , moderada, de 5 a $25 \%$ da superfície afetada; e 4 , severa, mais de $25 \%$ da superfície afetada. ${ }^{(2)}$ Médias seguidas por letras iguais, dentro de cada ano, maiúsculas nas colunas e minúsculas nas linhas, não diferem entre si, pelo teste de Tukey, a 5\% de probabilidade.

Tabela 5. Severidade do dano de estrias (1-4) $)^{(1)}$ em caqui 'Fuyu', após 70 dias de armazenagem em atmosfera modificada a $0^{\circ} \mathrm{C}$, mais sete dias a $23^{\circ} \mathrm{C}$, em três anos de avaliação e diferentes intervalos de colheita (dias), após a aplicação do 1-metilciclopropeno em meio aquoso (1-MCPa;), em frutos tratados com 1-MCPa e 1-metilciclopropeno gasoso (1-MCPg) ${ }^{(2)}$.

\begin{tabular}{|c|c|c|c|c|c|c|c|}
\hline \multirow[t]{2}{*}{$\overline{\text { Ano }}$} & \multirow[t]{2}{*}{ Tratamento } & \multicolumn{4}{|c|}{ Dias após a aplicação de 1-MCPa } & \multirow[t]{2}{*}{ Média } & \multirow[t]{2}{*}{ Desvio-padrão } \\
\hline & & 1 & 7 & 14 & 21 & & \\
\hline \multirow{4}{*}{2008} & Controle & $1,7 \mathrm{Aa}$ & - & $1,4 \mathrm{Aa}$ & - & $1,5 \mathrm{~B}$ & 0,54 \\
\hline & $1-\mathrm{MCPa}$ & $1,6 \mathrm{Aa}$ & - & $1,5 \mathrm{Aa}$ & - & $1,6 \mathrm{AB}$ & 0,66 \\
\hline & $1-\mathrm{MCPg}$ & $1,6 \mathrm{Aa}$ & - & $1,8 \mathrm{Aa}$ & - & $1,7 \mathrm{~A}$ & 0,74 \\
\hline & 1-MCPa+1-MCPg & $1,9 \mathrm{Aa}$ & - & $1,6 \mathrm{Aa}$ & - & $1,7 \mathrm{AB}$ & 0,74 \\
\hline \multirow{4}{*}{2009} & Controle & $2,4 \mathrm{ABa}$ & $1,9 \mathrm{Aa}$ & $2,0 \mathrm{Aa}$ & $1,9 \mathrm{Ba}$ & $2,1 \mathrm{AB}$ & 0,73 \\
\hline & 1-MCPa & $2,7 \mathrm{Aa}$ & $2,0 \mathrm{Ab}$ & $2,1 \mathrm{Ab}$ & $2,2 \mathrm{ABb}$ & $2,2 \mathrm{~A}$ & 0,82 \\
\hline & $1-\mathrm{MCPg}$ & $2,1 \mathrm{BCa}$ & 2,1Aa & $2,2 \mathrm{Aa}$ & $2,5 \mathrm{Aa}$ & $2,2 \mathrm{~A}$ & 0,70 \\
\hline & 1-MCPa+1-MCPg & $1,9 \mathrm{Ca}$ & $1,9 \mathrm{Aa}$ & $2,0 \mathrm{Aa}$ & $2,1 \mathrm{ABa}$ & $2,0 \mathrm{~B}$ & 0,72 \\
\hline \multirow{4}{*}{2010} & Controle & $2,1 \mathrm{Aa}$ & $1,4 \mathrm{Ab}$ & $1,9 \mathrm{Aa}$ & $1,5 \mathrm{Ab}$ & $1,7 \mathrm{~A}$ & 0,51 \\
\hline & 1-MCPa & $1,5 \mathrm{Bb}$ & $1,4 \mathrm{Ab}$ & $1,9 \mathrm{Aa}$ & $1,6 \mathrm{Ab}$ & $1,6 \mathrm{~A}$ & 0,52 \\
\hline & $1-\mathrm{MCPg}$ & $1,4 \mathrm{Bb}$ & $1,4 \mathrm{Ab}$ & 2,0Aa & $1,5 \mathrm{Ab}$ & $1,6 \mathrm{~A}$ & 0,51 \\
\hline & 1-MCPa+1-MCPg & $1,3 \mathrm{Bb}$ & $1,4 \mathrm{Ab}$ & $1,9 \mathrm{Aa}$ & $1,7 \mathrm{Aa}$ & $1,6 \mathrm{~A}$ & 0,51 \\
\hline
\end{tabular}

${ }^{(1)}$ Severidade do dano de estrias: 1 , ausência de dano; 2 , leve, menos de $5 \%$ da superfície afetada; 3 , moderada, de 5 a $25 \%$ da superfície afetada; e 4 , severa, mais de $25 \%$ da superfície afetada. ${ }^{(2)}$ Médias seguidas por letras iguais, dentro de cada ano, maiúsculas nas colunas e minúsculas nas linhas, não diferem entre si, pelo teste de Tukey, a $5 \%$ de probabilidade. 
Tabela 6. Severidade do dano de pinta preta (1-4) $)^{(1)}$ em caqui 'Fuyu', após 70 dias de armazenagem em atmosfera modificada a $0^{\circ} \mathrm{C}$, mais sete dias a $23^{\circ} \mathrm{C}$, em três anos de avaliação e diferentes intervalos de colheita (dias), após a aplicação do 1-metilciclopropeno em meio aquoso (1-MCPa), em frutos tratados com 1-MCPa e 1-metilciclopropeno gasoso (1-MCPg) ${ }^{(2)}$.

\begin{tabular}{|c|c|c|c|c|c|c|c|}
\hline \multirow[t]{2}{*}{ Ano } & \multirow[t]{2}{*}{ Tratamento } & \multicolumn{4}{|c|}{ Dias após a aplicação de 1-MCPa } & \multirow[t]{2}{*}{ Média } & \multirow[t]{2}{*}{ Desvio-padrão } \\
\hline & & 1 & 7 & 14 & 21 & & \\
\hline \multirow{4}{*}{2008} & Controle & $1,0 \mathrm{Bb}$ & - & $1,5 \mathrm{Ba}$ & - & $1,3 \mathrm{C}$ & 0,54 \\
\hline & 1-MCPa & $1,1 \mathrm{Bb}$ & - & $1,4 \mathrm{Ba}$ & - & $1,3 \mathrm{C}$ & 0,54 \\
\hline & 1-MCPg & $1,1 \mathrm{Bb}$ & - & $2,0 \mathrm{Aa}$ & - & $1,5 \mathrm{~B}$ & 0,63 \\
\hline & 1-MCPa+1-MCPg & $2,1 \mathrm{Ab}$ & - & $1,5 \mathrm{Bb}$ & - & $1,8 \mathrm{~A}$ & 0,70 \\
\hline \multirow{4}{*}{2009} & Controle & $2,1 \mathrm{Aa}$ & $2,1 \mathrm{Bab}$ & $1,5 \mathrm{Bc}$ & $1,7 \mathrm{Bbc}$ & $1,9 \mathrm{~B}$ & 0,78 \\
\hline & $1-\mathrm{MCPa}$ & $2,3 \mathrm{Aab}$ & $2,3 \mathrm{ABab}$ & $2,2 \mathrm{Ab}$ & $2,7 \mathrm{Aa}$ & $2,3 \mathrm{~A}$ & 0,78 \\
\hline & $1-\mathrm{MCPg}$ & $2,4 \mathrm{Aa}$ & $2,6 \mathrm{Aa}$ & $1,9 \mathrm{ABb}$ & $2,5 \mathrm{Aa}$ & $2,3 \mathrm{~A}$ & 0,70 \\
\hline & 1-MCPa+1-MCPg & $2,2 \mathrm{Aa}$ & $2,3 \mathrm{ABa}$ & $1,8 \mathrm{ABb}$ & $2,6 \mathrm{Aa}$ & $2,3 \mathrm{~A}$ & 0,79 \\
\hline \multirow{4}{*}{2010} & Controle & $1,3 \mathrm{Aa}$ & $1,3 \mathrm{Aa}$ & $1,3 \mathrm{Aa}$ & $1,5 \mathrm{Aa}$ & $1,3 \mathrm{~A}$ & 0,47 \\
\hline & 1-MCPa & $1,3 \mathrm{Aa}$ & $1,4 \mathrm{Aa}$ & $1,5 \mathrm{Aa}$ & $1,5 \mathrm{Aa}$ & $1,4 \mathrm{~A}$ & 0,49 \\
\hline & $1-\mathrm{MCPg}$ & $1,1 \mathrm{Ac}$ & $1,3 \mathrm{Abc}$ & $1,4 \mathrm{Abc}$ & $1,5 \mathrm{Aab}$ & $1,3 \mathrm{~A}$ & 0,46 \\
\hline & 1-MCPa+1-MCPg & $1,1 \mathrm{Ab}$ & $1,4 \mathrm{Ab}$ & $1,4 \mathrm{Ab}$ & $1,7 \mathrm{Aa}$ & $1,4 \mathrm{~A}$ & 0,48 \\
\hline
\end{tabular}

${ }^{(1)}$ Severidade do dano de pinta preta: 1 , ausência de dano; 2 , leve, menos de $5 \%$ da superfície afetada; 3 , moderada, de 5 a $25 \%$ da superfície afetada; e 4 , severa, mais de $25 \%$ da superfície afetada. ${ }^{(2)}$ Médias seguidas por letras iguais, dentro de cada ano, maiúsculas nas colunas e minúsculas nas linhas, não diferem entre si, pelo teste de Tukey, a 5\% de probabilidade.

inicial de 1-MCPa pulverizado às plantas $(150 \mathrm{mg}$ $\left.\mathrm{L}^{-1}\right)$ foi muito superior à concentração de $1-\mathrm{MCPg}$, na atmosfera de tratamento pós-colheita $\left(0,6 \mu \mathrm{L} \mathrm{L}^{-1}\right)$. Essa menor eficiência do tratamento pré-colheita com 1-MCPa, em comparação ao 1-MCPg, com base na quantidade de 1-MCP empregada, decorreu em grande parte da evaporação de 1-MCP, durante sua mistura em água no tanque de pulverização e durante a pulverização nas plantas, antes de atingir os frutos. Ademais, a penetração de 1-MCPa no fruto é reduzida pela menor difusão do 1-MCP em água e pela obstrução das lenticelas pela água (Argenta et al., 2007). Finalmente, diferenças quanto à eficiência do $1-\mathrm{MCPa}$ e $1-\mathrm{MCPg}$ podem estar relacionadas ao fato de que alguns dos receptores de etileno inibidos pelo 1-MCPg podem não ser inibidos pelo 1-MCPa, conforme mostrado em maçãs (Varanasi et al., 2013).

No presente estudo, a pulverização de 1-MCPa nos caquizeiros, no período de colheita comercial, preveniu a deterioração dos frutos durante a armazenagem refrigerada em AM. Além disso, a eficácia desse tratamento foi semelhante à do tratamento com 1-MCPg em pós-colheita, em frutos colhidos um dia após a pulverização do $1-\mathrm{MCPa}$, e a eficiência do 1-MCPa foi marcadamente reduzida com o atraso da colheita dos frutos a partir da data de sua pulverização.

\section{Conclusões}

1. A evolução da maturação de caqui (Diospyros kaki) 'Fuyu' na planta não é alterada pelo tratamento com 1-MCPa.

2. O tratamento com 1-MCPa reduz o amolecimento de polpa e a manifestação do distúrbio mancha translúcida após a colheita, porém, os benefícios diminuem com o atraso da colheita a partir da data da sua aplicação.

3. O tratamento 1-MCPa+1-MCPg não melhora a conservação da qualidade dos frutos, em comparação à aplicação isolada do $1-\mathrm{MCPg}$, mas as perdas dos benefícios do 1-MCPa com o atraso da colheita são compensadas pela aplicação do 1-MCPg.

4. A aplicação de 1-MCPa e 1-MCPg não tem efeito sobre a ocorrência dos distúrbios estrias e pinta preta.

\section{Agradecimentos}

À Coordenação de Aperfeiçoamento de Pessoal de Nível Superior (Capes), por bolsa concedida; à Fundação de Apoio à Pesquisa Científica e Tecnológica do Estado de Santa Catarina (Fapesc), pelo apoio financeiro; e à empresa AgroFresh, pelo fornecimento das formulações de 1-MCP. 


\section{Referências}

ARGENTA, L.C.; FAN, X.; MATTHEIS, J.P. Responses of 'Golden Delicious' apples to 1-MCP applied in air or water. HortScience, v.42, p.1651-1655, 2007.

ARGENTA, L.C.; VIEIRA, M.J.; SCOLARO, A.M.T. Conservação da qualidade de caqui 'Fuyu' em ambiente refrigerado pela combinação de 1-MCP e atmosfera modificada. Revista Brasileira de Fruticultura, v.31, p.323-333, 2009. DOI: 10.1590/ S0100-29452009000200006.

BEN-ARIE, R.; ZUTKHI, Y. Extending the storage life of 'Fuyu' persimmon by modified-atmosphere packaging. HortScience, v.27, p.811-813, 1992.

BITON, E.; KOBILER, I.; FEYGENBERG, O.; YAARI, M.; KAPLUNOV, T.; ACKERMAN, M.; FRIEDMAN, H.; PRUSKY, D. The mechanism of differential susceptibility to Alternaria black spot, caused by Alternaria alternata, of stem- and bottom-end tissues of persimmon fruit. Postharvest Biology and Technology, v.94, p.74-81, 2014. DOI: 10.1016/j.postharvbio.2014.03.008.

COLLINS, R.J.; TISDELL, J.S. The influence of storage time and temperature on chilling injury in Fuyu and Suruga persimmon (Diospyros kaki L.) grown in subtropical Australia. Postharvest Biology and Technology, v.6, p.149-157, 1995. DOI: 10.1016/0925-5214(94)00046-U.

CRISOSTO, C.H.; MITCHAM, E.J.; KADER, A.A. Recommendations for maintaining postharvest quality of horticultural commodities. Davis: University of California, 1999.

DEELL, J.R.; EHSANI-MOGHADDAM, B. Preharvest 1-methylcyclopropene treatment reduces soft scald in 'Honeycrisp' apples during storage. HortScience, v.45, p.414-417, 2010.

ELFVING, D.C.; DRAKE, S.R; REED, A.N.; VISSER, D.B. Preharvest applications of sprayable 1-methylcyclopropene in the orchard for management of apple harvest and postharvest condition. HortScience, v.42, p.1192-1199, 2007.

KRAMMES, J.G.; ARGENTA, L.C.; VIEIRA, M.J. Controle da maturação e conservação da qualidade pós-colheita de caqui 'Fuyu' pelo manejo do etileno. Revista Brasileira de Fruticultura, v.27, p.360-365, 2005. DOI: 10.1590/S0100-29452005000300006.

KRAMMES, J.G.; ARGENTA, L.C.; VIEIRA, M.J. Influences of 1-methylcyclopropene on quality of persimmon fruit cv. 'Fuyu' after cold storage. Acta Horticulturae, v.727, p.513-518, 2006. DOI: 10.17660/ActaHortic.2006.727.63.

KRAMMES, J.G.; ARGENTA, L.C.; VIEIRA, M.J.; BACARIN, M.A. Estabelecimento de índices de maturação para o ponto de colheita de frutos de caqui 'Fuyu'. Revista Agropecuária Catarinense, v.20, p.62-66, 2007.

MACRAE, E.A. Development of chilling injury in New Zealand grown 'Fuyu' persimmon during storage. New Zealand Journal of Experimental Agriculture, v.15, p.333-344, 1987. DOI: 10.1080/03015521.1987.10425579.

MANRÍQUEZ, D.; DEFILIPPI, B.G. Could preharvest application of 1-MCP improve postharvest life of 'Hayward' kiwifruit? Acta Hoticulturae, v.913, p.595-602, 2011. DOI: 10.17660/ ActaHortic.2011.913.81.

MCARTNEY, S.; OBERMILLER, J.D.; SCHUPP, J.R,; PARKER, M.L.; EDGINGTON, T.B. Preharvest 1-methylcyclopropene delays fruit maturity and reduces softening superficial scald of apples during long-term storage. HortScience, v.43, p.366-371, 2008.

NEUWALD, D.A.; GIEHL, R.F.H.; SESTARI, I.; BRACKMANN, A. Avaliação de filmes de polietileno para a conservação de caqui 'Fuyu' sob refrigeração. Revista Brasileira de Agrociência, v.11, p.95-99, 2005.

PIRRUNG, M.C.; BLEECKER, A.B.; INOUNE, Y.; RODRIGUEZ, F.I.; SUGAWARA, N.; WADA, T.; ZOU, Y.; BINDER, B.M. Ethylene receptor antagonists: strained alkenes are necessary but not sufficient. Chemistry and Biology, v.15, p.313-321, 2008. DOI: 10.1016/j.chembiol.2008.02.018.

SALVADOR, A.; ARNAL, L.; MONTERDE, A.; CUQUERELLA, J. Reduction of chilling injury symptoms in persimmon fruit cv. 'Rojo Brillante' by 1-MCP. Postharvest Biology and Technology, v.33, p.285-291, 2004. DOI: 10.1016/j.postharvbio.2004.03.005.

SCOLARO, A.M.T.; ARGENTA, L.C.; AMARANTE, C.V.T. do; PETRI, J.L.; HAWERROTH, F.J. Controle da maturação pré-colheita de maçãs 'Royal Gala' pela inibição da ação ou síntese do etileno. Revista Brasileira de Fruticultura, v.37, p.38-47, 2015. DOI: 10.1590/0100-2945-010/14.

SISLER, E.C.; SEREK, M. Inhibitors of ethylene responses in plants at the receptor level: recent developments. Physiologia Plantarum, v.100, p.577-582, 1997. DOI: 10.1111/ j.1399-3054.1997.tb03063.x.

VARANASI, V.; SHIN, S.; JOHNSON, F.; MATTHEIS, J.P.; ZHU, Y. Differential suppression of ethylene biosynthesis and receptor genes in 'Golden Delicious' apple by preharvest and postharvest 1-MCP treatments. Journal of Plant Growth Regulation, v.32, p.585-595, 2013. DOI: 10.1007/s00344-013-9326-8.

VILLALOBOS-ACUÑA, M.G.; BIASI, W.V.; FLORES, S.; MITCHAM, E.J.; ELKINS, R.B.; WILLITS, N.H. Preharvest application of 1-methylcyclopropene influences fruit drop and storage potential of 'Bartlett' pears. HortScience, v.45, p.610-616, 2010.

WATKINS, C.B. The use of 1-methylcyclopropene (1-MCP) on fruits and vegetables. Biotechnology Advances, v.24, p.389-409, 2006. DOI: 10.1016/j.biotechadv.2006.01.005.

YUAN, R.; LI, J. Effect of sprayable 1-MCP, AVG, and NAA on ethylene biosynthesis, preharvest fruit drop, fruit maturity, and quality of 'Delicious' apples. HortScience, v.43, p.1454-1460, 2008.

Recebido em 12 de março de 2015 e aprovado em 4 de março de 2016 